\title{
Variability in management of symptomless microhaematuria in schoolchildren
}

\author{
Jochanan Benbassat, Mansour Gergawi, Martin Offringa, Jacob E Bearman, \\ Alfred Drukker
}

JDC Brookdale Institute, Jerusalem, Israel

J Benbassat

\section{Division of} Community Health, Faculty of Health Sciences, Ben-Gurion University, Beer-Sheva, Israel $M$ Gergawi JE Bearman

\section{Department of} Pediatrics, Emma Children's University Hospital, Academic Medical Centre, Amsterdam, The Netherlands M Offringa

Division of Pediatric Nephrology, Shaare Zedek Medical Centre and Hebrew

University-Hadassah Medical School, Jerusalem, Israel A Drukker

Correspondence to Benbassat MD, PO Box 3894, Jerusalem, 91037 , Israel

\begin{abstract}
Summary
The evaluation of incidentally detected symptomless microhaematuria in schoolchildren is controversial. Some authors advocate varying numbers of immediate tests, while others recommend investigations only in cases who develop systemic symptoms or signs, or a decline in renal function. The objective of this study was to estimate the extent to which this uncertainty affects the declared habits of practising physicians. A sample of 16 family physicians, 42 primary care paediatricians and 26 full-time hospital-based paediatric nephrologists in Israel were asked to complete a survey using a written case of a hypothetical eight-year-old boy with incidentally detected symptomless microhaematuria. Responses were received from $16(100 \%), 18(43 \%)$ and $18(69 \%)$, respectively. The mean number of requested tests, other than follow-up examination of the urine, were 1.5 (range 0-5) for family physicians, 2.5 (1-5) for primary care paediatricians and $5.3(2-12)$ for paediatric nephrologists, at an average cost of NIS 408 (US\$ 136), NIS 454 (US\$ 151) and NIS 860 (US\$286), respectively. There was also a marked variability within subspecialty groups, so that some family physicians recommended more tests at a higher cost than some of the paediatric nephrologists. There was a marked and unexplained variability within and among the three groups of respondents regarding the extent of the evaluation. The main reason for this variability is probably the uncertainty about the scientifically appropriate way to approach this condition in a symptomless child.
\end{abstract}

Keywords: haematuria; variability in clinical practice

Recognition of variation is fundamental in quality control, and industrial experts have already developed principles and techniques for quality improvement based on a rigorous analysis of variation in outcomes and processes. It has been suggested that these principles may also be applicable in healthcare. ${ }^{1}$ There are indeed marked, unexplained, variations between geographic areas in hospitalisation rates, expenditures for physicians' services and utilisation of surgical procedures. ${ }^{2}$ Some variability of clinical practice is certainly justified by the unique circumstances and preferences of individual patients; in most cases, however, analysis suggests that it results from variations in the rules of practice. Today, academic standards of care are compatible with widely varying patterns of practice. Wennberg ${ }^{2}$ pointed out that "the failure to evaluate the outcomes of surgical practice, diagnostic tests and the uses made of hospitals contrasts sharply with the careful evaluations mandated for drugs", and that this "double standard for truth in medicine compromises the rationality of medical decisions with significant, deleterious consequences for patients and the healthcare economy".

Most studies of unexplained variability in clinical practice have focused on surgical procedures, hospital utilisation and mortality. Studies of the variability in diagnostic approach are relatively rare. ${ }^{3-6}$ This paper deals with the variability in approach to incidentally detected symptomless microhaematuria (IMH) in schoolchildren, without growth failure, hypertension, oedema, proteinuria, casts in the urinary sediment, or impairment of glomerular function.

IMH is a frequent abnormality on urinary screening of schoolchildren ${ }^{7}$ and the second most common cause for referral to paediatric nephrology clinics in the US. ${ }^{8}$ Its point prevalence among schoolchildren is about $1 \% .^{79^{10}}$ There is general agreement that recurrent or persistent microhaematuria in an otherwise healthy child mandates a search for precipitating factors (infection, medications, bleeding tendency), a thorough urinalysis (protein, casts), and a baseline serum creatinine level. However, opinion is divided regarding the extent of additional evaluation of IMH. At one extreme, it is advocated not to perform further examinations unless the child develops systemic symptoms or signs, or a decline in renal function. ${ }^{7}$ At the other extreme, however, it has been argued that "... a screening program [for urinary abnormalities] may open the way for the early management [of glomerulonephritis]", ${ }^{11}$ thereby implicitly recommending a full investigation for every case of IMH. Even ignoring these two extreme recommendations, the number of immediate examinations recommended in textbooks and published reviews have varied between one and five at a cost between $\$ 46$ and $\$ 332$ (table 1 ). 
Table 1 Recommended approach to incidentally detected isolated microhaematuria, beyond follow-up examinations

\begin{tabular}{lll}
\hline Ref & Recommendation & Estimated cost $(\$)$ \\
\hline 12 & Renal sonography & 332 \\
& Urinary calcium/creatinine ratio \\
& Sickle cell preparation \\
Serum complement levels $(\mathrm{C} 3, \mathrm{C} 4)$ & \\
& ANA and anti-DNA. & \\
13 & Urinary calcium/creatinine ratio & 61 \\
14 & Urine culture & 144 \\
15 & Renal sonography & 46 \\
16 & Urine culture & 79 \\
& Urinary calcium/creatinine ratio & \\
\hline
\end{tabular}

*The inclusion of sickle cell preparation is recommended only for selected populations, and may be omitted if the patient has been screened before.

The objective of this paper is to estimate the extent to which this uncertainty has affected the declared habits of practising physicians. We hypothesized that a significant variability would be detected in the recommended approach of family physicians, ambulatory paediatricians and paediatric nephrologists to IMH.

\section{Methods}

\section{STUDY POPULATION}

The surveyed practitioners were family physicians, who participated in a continuing education programme in Beer Sheva $(n=16)$, primary care paediatricians in nine randomly selected out-patient facilities throughout Israel $(n=42)$, and full-time paediatric nephrologists in eight hospitals in Israel $(n=26)$. The continuing education programme did not include nephrology and was unrelated to the subject of the survey. The participants were asked to indicate the test(s) they would recommend for the case presented in box 1. The family physicians were approached personally, while the primary care paediatricians and paediatric nephrologists were contacted by mail, and reminded to respond during a professional meeting. Responses were received from 16 $(100 \%), 18(43 \%)$ and $18(69 \%)$, respectively.

\section{INSTRUMENT}

A self-administered questionnaire (box 1), consisting of a hypothetical case of IMH in an eight-year-old boy, was used in the study and returned without identification. The responders were asked to indicate the tests they would perform.

\section{VARIABLES}

Subspecialty training was the independent variable, whereas the dependent variables were the tests requested and their cost. The average number of recommended tests (other than follow-up urine examination), and their cost, was calculated per physician for each specialty group. The cost of the tests were obtained from the office of the Soroka Medical Centre (a public Sick Fund hospital) in Beer Sheva in new Israeli shekels (NIS; 3 NIS = 1 US dollar). For example, the cost of sonography of the urinary tract, determination of serum complement level, and kidney biopsy in 1996 was NIS

\section{Questionnaire}

David, aged eight years, is referred to you three weeks after a screening examination in his school detected 8-10 red blood cells (RBC) per high power field (HPF) in the urinary sediment. David was born after an uneventful pregnancy and delivery. There are no known relatives with renal disease or deafness. He had all vaccinations and his growth and development were normal. His parents deny any serious diseases in the past, present complaints or recent medication.

Examination reveals an alert intelligent boy without physical findings. There is no oedema, his blood pressure is $100 / 70 \mathrm{mmHg}$ and a repeated urinalysis reveals 4-6 RBC/HPF, but no protein or casts. Blood urea and creatinine are normal.

What tests would you recommend? (choose as many as you feel indicated):

- follow-up examination

- 24-h urinary calcium excretion

- calcium/creatinine ratio in urine

- 24-h urinary protein excretion

- creatinine clearance

- renal ultrasound

- intravenous pyelography

- renal scan (DTPA)

- renal scan (DMSA)

- referral to nephrologist for biopsy

- referral to urologist for cystoscopy

- other (add those you feel indicated)

Box 1

431 (US\$144), NIS 125 (US\$ 42) and NIS 1446 (US $\$ 482$ ), respectively.

\section{STATISTICAL ANALYSIS}

The significance of the differences between subspecialty groups was estimated by the Euclidean Distance Function (EDF) Statistics. ${ }^{17}$ Unlike the 'classical' statistical methods that use the mean and standard deviation, the EDF makes use of the median and average pair-wise deviations. Unlike the mean, the median is not affected by extreme outliers, and therefore its use avoids the usual distortions of the geometrical visualisation of the results of the least squares statistics.

\section{Results}

The mean number of requested tests, other than follow-up examination of the urine were 1.5 (range $0-5$ ) for family physicians, $2.5(1-5)$ for primary care paediatricians and $5.3(2-12)$ for paediatric nephrologists, at an average cost of NIS 408 (US $\$ 136$ ), NIS 454 (US $\$ 151$ ) and NIS 860 (US\$286), respectively ( $p<$ 0.0001 ) (table 2). There was also a marked variability within subspecialty groups, so that some family physicians recommended more tests at a higher cost than some of the paediatric nephrologists (table 2). Still, the respondents to this survey appeared to agree on several areas. Most of them favoured sonography, but not invasive examinations. None of the 52 respondents ordered an intravenous pyelography, a radionuclear scan of the kidneys or kidney biopsy. Urine cultures were recommended by three family physicians, only one respondent recommended urologic referral for 
Table 2 Recommendations of 16 family physicians, 18 ambulatory paediatricians and 18 hospital-based paediatric nephrologists for the management of isolated asymptomatic microhaematuria in an otherwise healthy eight-year-old child

\begin{tabular}{llll}
\hline Recommended tests & Family physicians & Ambulatory paediatricians & Paediatric nephrologists \\
\hline Number of tests & 1.5 & 2.5 & 5.3 \\
Average & $0-5$ & $1-5$ & $2-12$ \\
Range & 136 & 151 & 286 \\
Cost (US \$) & $0-258$ & $144-363$ & $158-420$ \\
Average & & & \\
Range & &
\end{tabular}

^Excluding follow-up examinations.

cystoscopy, and none recommended an examination of urinary red cell morphology (table 3).

\section{Discussion}

The presented findings should be interpreted with caution. The sample of family physicians was not random, but rather selected by convenience, and included only those attending a continuous education session. The low response rates of the paediatricians and nephrologists, although not unusual for a mail survey of physicians without telephone contact, reduces the confidence in the results. It is possible that only physicians who were comfortable with their own expertise responded to the survey. Responses to written scenarios may not reflect real-life behaviour, and they may not be generalisable over other geographic areas because of differences in the incidence of the various disorders, eg, hypercalciuria, that may present with IMH. In fact they may not be generalisable even over Israeli physicians. Therefore, the results of this survey should not be interpreted as suggesting that either of the reported approaches, eg, that recommended by the majority, or the least expensive one, is the best. In the absence of a 'gold standard' for the appropriate management of IMH, we are not in a position to identify the 'correct' one. The only conclusion derived from the presented findings is that they indicate a considerable variability within and among the groups of respondents regarding the extent of the evaluation of a hypothetical case of IMH.

There are several possible explanations of this variability. Conceivably, primary care physicians differ from hospital-based specialists in expectations for diagnostic certainty, extent of defensive clinical practice, or in the differential diagnosis they generate in response to the presented case. Hospital-based specialists commonly see selected patients who have passed

Table 3 Recommendations for the management of isolated asymptomatic microhaematuria in an otherwise healthy eight-year-old child. Figures are numbers recommending each option with the percentage in parentheses

\begin{tabular}{|c|c|c|c|}
\hline Recommended tests & Family physicians & $\begin{array}{l}\text { Ambulatory } \\
\text { paediatricians }\end{array}$ & Paediatric nephrologists \\
\hline Urinary calcium/creatinine ratio & $1(6)$ & $12(67)$ & $14(78)$ \\
\hline 24-h urinary calcium & - & $7(39)$ & $13(72)$ \\
\hline 24-h urinary protein & $2(13)$ & $5(28)$ & $4(22)$ \\
\hline Creatinine clearance & - & $3(17)$ & $4(22)$ \\
\hline Renal ultrasound & $12(75)$ & $16(89)$ & $18(100)$ \\
\hline Intravenous pyelogram & - & - & - \\
\hline Renal scan (DTPA) & - & - & - \\
\hline Renal scan (DMSA) & - & - & - \\
\hline Referral to nephrologist for biopsy & - & - & - \\
\hline Referral to urologist for cystoscopy & - & $1(6)$ & - \\
\hline \multicolumn{4}{|l|}{ Other $\star \star$ : } \\
\hline Urinalysis in family members & - & - & $1(6)$ \\
\hline Urinary specific gravity & - & - & $1(6)$ \\
\hline Urine culture & $3(19)$ & - & - \\
\hline Throat swab & $1(7)$ & - & - \\
\hline Urinary red cells after exercise & $1(7)$ & - & - \\
\hline Fundoscopy & - & - & $2(11)$ \\
\hline Audiometry & $1(7)$ & - & $2(11)$ \\
\hline Serum uric acid & - & - & $2(11)$ \\
\hline Serum calcium & - & - & $2(11)$ \\
\hline Serum phosphorus & - & - & $2(11)$ \\
\hline Fasting serum glucose & - & - & $1(6)$ \\
\hline $24-\mathrm{h}$ urinary uric acid & - & $1(6)$ & $5(28)$ \\
\hline 24-h urinary oxalate & - & - & $2(11)$ \\
\hline 24-h urinary cysteine & - & - & $1(6)$ \\
\hline ESR & $2(13)$ & - & - \\
\hline Serum complement & - & - & $8(44)$ \\
\hline ANF, LE preparation & - & - & $6(33)$ \\
\hline ASTO & - & - & $2(11)$ \\
\hline Serum immunoglobulins & - & - & $4(22)$ \\
\hline Blood coagulation studies & - & - & $1(6)$ \\
\hline Blood count & $1(7)$ & - & $1(6)$ \\
\hline No tests ordered & $4(25)$ & - & - \\
\hline
\end{tabular}

*This option was excluded from the questionnaire mailed to paediatric nephrologists and replaced by renal biopsy.

$\star \star$ These 'other' recommendations did not appear in the questionnaire, but were added by the responders. 


\section{Summary/learning points}

- recognition of unexplained variation in clinical practice is fundamental in its quality control. Some of this variability is justified by the unique circumstances and preferences of individual patients. In most cases, however, it results from variations in the rules of practice

- IMH is a frequent abnormality on urinary screening of schoolchildren and the second most common cause for referral to paediatric nephrology clinics in the US

- a survey conducted by the authors among Israeli physicians revealed a considerable variability in the recommendations for evaluation of a hypothetical case of an eight-year-old boy with IMH, both between and within specialties. The mean number of recommended tests was 1.5 (range 0-5) for family physicians, 2.5 (1-5) for primary care paediatricians and $5.3(2-12)$ for paediatric nephrologists

- the main reason for the observed variability in physicians' management of IMH in a symptomless child is probably the uncertainty about the scientifically appropriate approach to such cases. The number of immediate tests recommended in textbooks and published reviews have varied between one and five, at a cost between $\$ 46$ and $\$ 332$

- the uncertainty regarding rules of clinical practice is not inevitable. The identification of unexplained differences in recommended or actual practice, should be followed by their analysis and formulation of agreed guidelines

\section{Box 2}

the filter of referral by the primary care physician. These patients probably have recurrent

1 Laffel G, Blumenthal D. The case for using industrial quality management science in health care organizations. $\mathscr{f} A M A$ 1989;262:2869-73.

2 Wennberg JE. Unwanted variations in the rules of practice. ҰAMA 1991;265:1306-7.

3 Barneveld-Binkhuysen FH, Puylaert CB. Influence of CT on radiologic utilization in the Netherlands. Radiology 1988 ; 169:827-9.

4 Khatri K, Kaufman R, Baigelman W. Utilization of pulmonary function tests by primary care internists in a community hospital. Am $\mathcal{F}$ Med Qual 1994;9:49-53.

5 Kingston ME. Management of anemia in a general city hospital: value of chart review in establishing deficiencies in care. Can Med Assoc $\mathcal{F}$ 1978;118:50-2.

6 Vink R, Offringa M, van der Does E. The approach of family physicians to febrile convulsions (Dutch). Huisarts Wet 1990;33:263-7.

7 Dodge WF, West EF, Smith EH, Bruce-Harvey T. Proteinuria and hematuria in schoolchildren: epidemiology and early natural history. $₹$ Pediatr $1976 ; 88: 327-47$.

8 Foreman JW, Chan JC. 10-year survey of referrals to a pediatric nephrology program. Child Nephrol Urol 1990;10:8-13.

9 Vehaskari VM, Rapola J, Koskomies O, Vilska J, Hallman N. Microscopic hematuria in school children. Epidemiology and clinicopathological evaluation. $\mathcal{f}$ Pediatr 1979;95:67684 . rather than transient haematuria, with more severe findings than isolated microhaematuria. The likelihood of serious disorders in such patients is higher than in those seen in the primary care setting. Differences in perceived expectations for diagnostic certainty, in the extent of defensive clinical practice, or in the differential diagnosis generated in children with IMH probably explain also the marked variability within the three groups of respondents. However, we believe that the main reason for this variability is the uncertainty reflected in the different recommendations in textbooks and published reviews (table 1) about the approach to IMH.

This uncertainty is not inevitable. ${ }^{2}$ The identification of unexplained differences in practice, whether those recommended in texts and reviews, or within and between groups of physicians, should be followed by their analysis and a formulation of agreed guidelines. ${ }^{18}$ The findings of this survey identify the need for such guidelines in the approach to IMH in children. Recently, we explored the likely causes for IMH among children as a first step towards the identification of the most sensible approach to this common condition and identified three possible strategies that are consistent with presently available data: no tests, sonography alone, or sonography and serum $\mathrm{C}_{3}$ levels. ${ }^{19}$ Hopefully, the choice between these three strategies will be resolved by additional observational studies of children with IMH, or by formal decision analysis.

Supported by a grant from the Chief Scientist, Ministry of Health, Jerusalem

10 Hisano S, Kawano M, Hatae K, et al. Asymptomatic isolated microhaematuria: natural history of 136 children. Pediatr Nephrol 1991;5:578-81.

11 Kitagawa T. Lessons learned from the Japanese nephritis screening study. Pediatr Nephrol 1988;2:256-63.

12 Vehaskari VM. Ask the expert. Pediatr Nephrol 1989;3:32.

13 Edelman CM, ed. Pediatric kidney disease, 2nd edn. Boston: Little Brown Co, 1991.

14 Lieu TA, Grasmeder HM, Kaplan BS. An approach to the evaluation and treatment of microscopic hematuria. Pediatr Clin North Am 1991;38:579-92.

15 Rudolph AM, ed. Rudolph's pediatrics, 19th edn. Norwalk: Appleton \& Lange, 1991.

16 Fitzwater DS, Wyatt RJ. Hematuria. Pediatr Rev 1994;15: 102-5.

17 Mielke PW. Non-metric statistical analysis: some metric alternatives. $\mathcal{F}$ Stat Plan Infer 1986;13:377-87.

18 Donabedian A. Institutional and professional responsibilities in quality assurance. Qual Assur Health Care 1989;1:311 .

19 Benbassat J, Gergawi M, Offringa M, Drukker A. Symptomless microhaematuria in schoolchildren. Causes for the variability in recommended management strategies. $Q \mathcal{F} M e d$ 1996;89:845-54. 